\title{
Evaluation of Public Servant Execution Based on Data Mining Technique and Multiple Factors Joint Modeling Analysis
}

\author{
Yang $\mathrm{Du}^{1,2}$, Wenbin Chen ${ }^{1}$ and Di Cheng ${ }^{2}$ \\ ${ }^{1}$ School of Marxism, Northeast Forestry University, Harbin, China \\ ${ }^{2}$ College of Humanities and Law, Northeast Agricultural University, Harbin, China \\ E-mail: ${ }^{a}$ doyoung2008@163.com; ${ }^{b}$ wenbinchen@263.net; ${ }^{c}$ chengdi100@sina.com
}

\begin{abstract}
With the rapid development of computer science and technology, data mining modelling techniques have emerged and rapidly developed as an alternative powerful meta-learning tool to accurately and fast analyze the massive volume of data generated by modern applications. The combination of data analysis technique and evaluation of public servant execution is urgently needed. Improve the execution of public servants at the grass-roots level is one of the important link to strengthen the construction of authority administrative efficiency of administrative goals is very important. Enhance the execution must first cultivate advanced concept, armed with advanced execution concept to the vast number of public servants at the grass-roots level. The assessment of public execution has a lot of traditional methods and models can be used but there is limitation. The limitation could be concluded as the following. Carelessness or poor sensitivity, At the grassroots level, the implementation of the main body of the general public servants at the grass-roots level and they can perform in place, one of the important factor is whether the leader on the work division of labor, organization, management and supervision effectively. In this paper, we conduct research on evaluation of public servant execution based on data mining technique and joint modeling analysis of multiple factors under big data environment. Firstly, we introduce some state-of-the-art clustering algorithm to serve as the basis of our model. Combined with deep neural network and optimization modelling, we propose our support vector machine based data clustering algorithm through multiple factor modelling. Subsequently, we discuss the principles on evaluation of public servant execution and process management. In the experimental part, we conduct experiment on both data clustering based data pre-processing step and the evaluation of elements' weight for process management. The result indicates the most important factor for management and the feasibility and effectiveness of our proposed clustering method. Future potential research areas are also discussed in the final Section.
\end{abstract}

Keywords: Evaluation of Public Servant Execution, Data Mining Technique, multiple Factors Joint Modeling Analysis

\section{Introduction}

As the core algorithm of data mining technique, machine learning methodology can be broadly classified into transductive or inductive approach, respectively. In the transformation type study, objective is to learn to observe, specific examples to specific examples from the same distribution. In contrast, induction from observational learning training situation will study the real distribution of a set of assumptions test cases. Conversion ratio induction, induction need to solve the problem of a more general (assuming the real distribution) to solve problems in a more specific issue. Modern applications of data mining techniques could be divided into the following parts: (1) Data mining for pattern recognition. Through mining the captured data, we could perceive the pattern of the received object, such as image, video and document. In [1], Anyela et al. 
proposed an objective definition of rosette shape variation using a joint image processing and data mining approach. In [2], Vishwanath et al. proposed a $\mathrm{KNN}$ based machine learning approach for text and document mining and they adopt the data mining technique to polish the current simple models of document analysis and enhance the accuracy of detecting the specific blocks of words. (2) Data mining for business. With the popularity of Internet and computer, online data is bursting which is essential for companies which may help analysis the preference of the customer and response of the market. In [3], Erman et al. conducted research on predicting dropout student through mining the online education program's data. In order to classify dropout students, four data mining approaches were applied based on k-Nearest Neighbour, Decision Tree, Naive Bayes and Neural Network. In [4], Witte introduced a novel perspective of data mining, inference and prediction in the age of big data. (3) Data mining for management and evaluation of public servant execution. In [5], Xu's group conducted research on improving management of aquatic invasions by integrating shipping network, ecological, and environmental data. The combination of data mining and management is challenging due to its complex mechanism. There are more related researches on data mining and analysis in the literature review [6-15].

Research on evaluation of public servant execution is important for our society and government. Public servants at the grass-roots level to stand in the forefront of implementation of the party and the state of various policies, the first line, directly to the masses, service masses which is the bridge connecting state and the masses and the execution of the quality of the relationship between the party and national policy implementation and effects. We need to overcome the problems of execution of public servants at the grass-roots level to strive to find the effective ways to improve execution so as to continuously enhance administrative efficiency and obtain better service to the masses. With higher execution, citizens will benefit more not only related to the efficient work but also the high-quality life. In the process of realize the goal of policy, the government executive ability is the key to the effect to translate into action the administrative goal and execution of public servants at the grass-roots level about the final and effective realization of the goal administration. Under the big data environment, modelling execution through multiple factors is a feasible and applicable way to gain higher accuracy.

The traditional methods could be summarized as the follows. (1) Cultivate the advanced idea of execution. Concept is the premise and basis of action. Improve the execution, the execution of must first cultivate advanced idea, only to the mind of the grass-roots public servants armed with advanced execution concept, strengthen its political consciousness and the objective consciousness, responsibility consciousness and the general situation consciousness which make it become the source of their conscious awareness and motivation. (2) Build efficient execution ability. To improve the execution of grass-roots public servants, we should focus on enhancing its ability quality. One method is the ability to implement responsibility of the quality. Public servants at the grass-roots level to strengthen political theory knowledge learning at the same time which also must keep pace with the times, strengthen the market economy, science and technology, laws and regulations and management and other related knowledge. (3) Create efficient enforcement mechanism. Establish and improve the efficient enforcement mechanism, is to improve the security of the execution of the public servants at the grass-roots level. Science define and refine their responsibility to accrual of proportion as the criterion, build system of post responsibility management by objectives and responsibility, do to post proportional amount, responsibility to the people. However, the traditional methods have not focus on the data obtained from the real world survey. Combining the advances of the big data and information technology into the research is urgently needed.

In this paper, we conduct research on evaluation of public servant execution based on data mining technique and joint modeling analysis of multiple factors under big data 
environment. In the data mining procedure, we adopt the principle technique of neural network (NN) and quantum support vector machine (QSVM) to classify the data into clusters, and later, we extract the features learned from the clusters to undertake the task of evaluating execution of public servants. The experimental result shows that our methodology could correctly classify the levels of public servants into three layers: highefficient, medium and low-efficient with high accuracy. The paper could be organized as the follows: In the Section 2, we introduce the overview of popular adopted data classification algorithms under the big data environment. Based on this chapter, we propose our methodology in the Section 3. In the Section 4, we discuss the principles on evaluation of public servant execution and process management to combine the evaluation with data analysis and modelling. In the Section 5, we conduct experimental analysis on data pre-processing and measurement result, the result indicates that our preprocessing algorithm based on the proposed fuzzy clustering method outperforms the other state-of-the-art approaches. In the final Section, we give the conclusion and the prospect.

\section{Two Traditional Data Classification Algorithms Frequently Used by Evaluation of Public Servant Execution}

In this Section, we introduce two state-of-the-art and important clustering algorithms with detailed discussion. The selected ones are: FCM and EM algorithms. These two models serve as the basic of our proposed method and we will conduct comparison experiment in the following chapters.

\subsection{Fuzzy C-means Algorithm (FCM)}

FCM [16] is a representative algorithm of fuzzy clustering which is based on K-means concepts to partition dataset into clusters. The FCM algorithm is a "soft" clustering method in which the objects are assigned to the clusters with a degree of belief. Fuzzy cmeans algorithm aims at minimizing the intra-cluster variance. It inherited problem of $\mathrm{k}$ means, however, it is a local minimum and finally a cluster depends on the choice of initial weights. The objective function for FCM I defined in the formula 1:

$$
J=\sum_{i=1}^{n} \sum_{k=1}^{c} \mu_{i k}^{m}\left|p_{i}-v_{k}\right|^{2}
$$

Where $J$ represents the objective function, $n$ is the number of objects, $c$ is the number of clusters, $\mu_{i k}^{m}$ is the likelihood values. The pseudo-code for FCM is shown in the Table 1.

Table 1.The FCM Algorithm

Algorithm 1. Fuzzy C-means Algorithm (FCM )
1. Input: Given the dataset desire number of clusters,
fuzzy parameters and stopping condition.
2. Output: A list of c cluster centers and a partition matrix are produced.
3. Calculate the cluster centroids and the objective value.
4. Compute the membership values stored in the matrix.
5. If the objective value of between consecutive iterations is less than
the stopping condition, then stop = true.
6. While (!stop).

The FCM algorithm is an unsupervised learning process and implementation is relatively simple and fast calculation speed, the objective function is the Euclidean distance which has intuitive geometric meaning. However, like other clustering algorithms, 
FCM algorithm has its own disadvantages and applicable object, first of all, the FCM clustering algorithm only class center to represent the class, so only suiTable for find ball type, etc. The convex shape of the clusters and in many cases, the algorithm is sensitive to noise data. Secondly the algorithm have two parameters before clustering analysis requirements given advance appropriate assignment, namely fuzzy weighted index and clustering number and this will affect the analysis of effect of FCM algorithm. Finally the algorithm is sensitive to initialize the classification parameters, the division of different initialization may get different results, also is more sensitive to noise data. Due to the mentioned drawbacks, we decide not to use the algorithm.

\subsection{The Expectation-maximization (EM) Algorithm}

Expectation-maximization algorithm [17] is generally designed to estimate the maximum likelihood parameters of a statistical model in many situations, such as the one where the equations cannot be solved directly. In the Table 2, we introduce the basic expectation-maximization algorithm.

Table 2. The Expectation-Maximization Algorithm

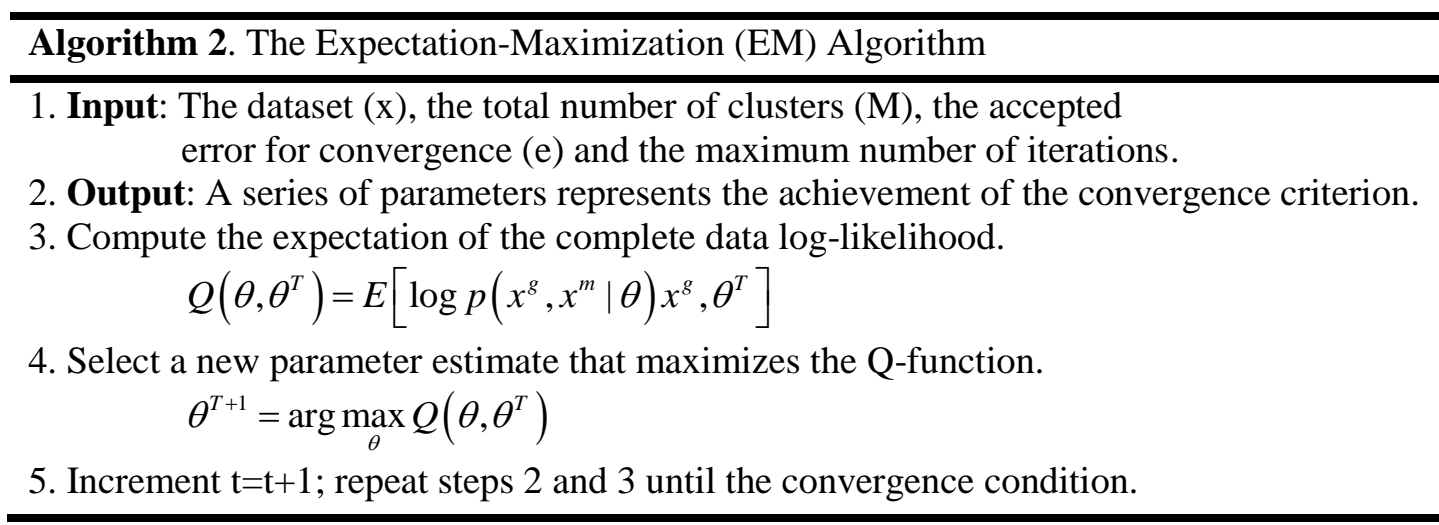

In view of the defects of FCM algorithm and characteristics, people try various methods to try to optimize the FCM algorithm, the FCM algorithm to get the most reasonable and efficient practical use. EM algorithm in one of the most famous algorithms adopted. However, it is also sensitive to noise which makes us not choose this method.

\section{Our Proposed Methodology for Evaluation of Public Servant Execution under the Big Data Environment}

\subsection{Principles of Support Vector Machine}

The task for the SVM is to classify a vector into one of two classes. The form of training data is shown in the formula 2.

$$
\left\{\left(\vec{x}_{j}, y_{j}\right): \vec{x}_{j} \in \square^{N}, y_{j}= \pm 1\right\}_{j=1,2, \ldots, M}
$$

Where, $y_{j}$ is depending on the belonging class. For the classification, the SVM finds a maximum-margin hyperplane with normal vector $\vec{w}$ that divides the two classes. The margin is given by two parallel hyperplanes that are separated by the maximum possible distance $2 /|\vec{w}|$ with no data points inside the margin. The dual formulation for the issue is 
maximizing over the Karush-Kuhn-Tucker multipliers (KKTM) $\vec{\alpha}=\left(\alpha_{1}, \ldots, \alpha_{M}\right)^{T}$ the function:

$$
L(\vec{\alpha})=\sum_{j=1}^{M} y_{j} \alpha_{j}-\frac{1}{2} \sum_{j, k=1}^{M} \alpha_{j} K_{j k} \alpha_{k}
$$

The hyperplane parameters are recovered from $\vec{w}=\sum_{j=1}^{M} \alpha_{j} \vec{x}_{j}$ and $b=y_{j}-\vec{w} \cdot \vec{x}_{j}$. The kernel functions for SVM can be found easily in the literature reviews [18-25]. The formula 4 is a sample kernel function.

$$
K_{j k}=k\left(\vec{x}_{j}, \vec{x}_{k}\right)=\vec{x}_{j} \cdot \vec{x}_{k}
$$

\subsection{Deep Neural Network Combined Model}

In the last few years, Deep Neural Networks have replaced Gaussian Mixture Models as the state-of-the-art acoustic model. DNN optimization based on a Cross-Entropy (CE) loss function reflecting classification of temporally local speech frames into context-dependent states for using in a "hybrid" DNN/HMM system is a simple, easily implemented approach used successfully in several studies [26-30]. Although simple, but the frame discriminative training is the ultimate goal of cannot guarantee optimization, precision in the whole discourse, decoding and a complete model of the language. The description of the first paragraph optimization is using utterance-level maximum mutual information (MMI) standard merge recognition. The flowchart could be seen in the Figure 1.

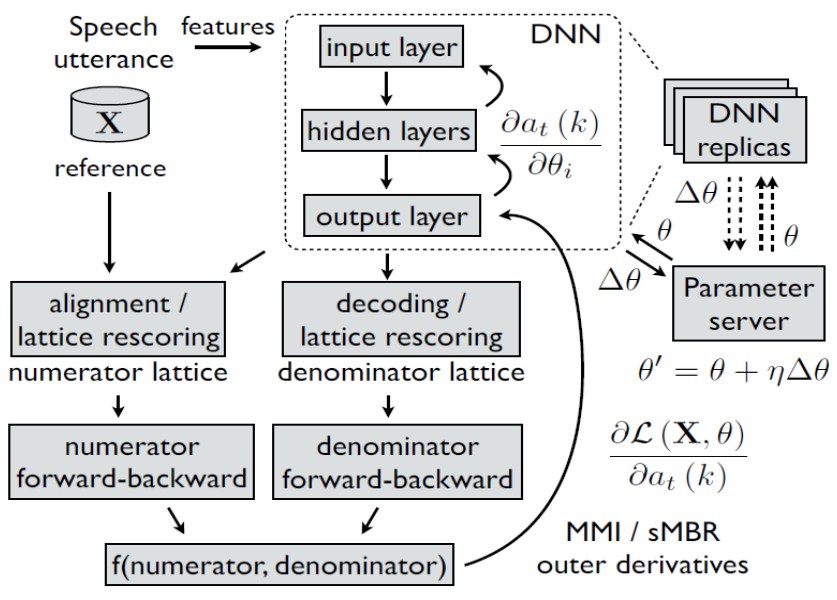

Figure 1.The Explanation of DNN

Though the effectiveness of SGD has been known for decades, schemes to scale it via parallelization have run into the fundamental problem of memory-locking and synchronous model updates, which drastically slows down learning performance. The key features are: (1) A parameter server that (1) Holds a snapshot of the current parameter set, which it can communicate on request; (2) Updates the parameter set, given incoming parameter gradients and a learning rule; (2) A set of model replicas that (1) Make requests to the parameter server to obtain the latest version of the parameter set; (2) Update a local representation of the cost function gradient according to their local parameter set and the data shard being processed; (3) Communicate their local gradient back to the parameter server. Copy do not wait for the latest version update, on the contrary, they calculate derivatives using the model, is usually out of date. Asynchronous allows faster optimization, divergence if the parameter update the risk is too big. 


\subsection{Our Proposed Optimization Method}

In the following, different optimization criteria and their outer derivatives are briefly summarized. (1) Frame-level Cross-Entropy. In the framework of this study is a simple baseline level CE optimization criterion, promote the past most of the GMM recognition precision of baseline.

$$
L_{\text {XENT }}(X, \theta)=\sum_{t=1}^{T} \sum_{k=1}^{N} y_{t}(k) \log \frac{y_{t}(k)}{y_{t}(k)}
$$

The outer derivative is shown in the formula 6.

$$
\frac{\partial L_{X E N T}(X, \theta)}{\partial a_{t}(k)}=y_{t}(k)-y_{t}(k)
$$

(2) Maximum Mutual Information (MMI) for sequence training. For DNNs, the loglikelihood for $\mathrm{X}$ gives a word string $S_{j}$ with best Viterbi state sequence which is formulated as the equation 7 .

$$
\log p\left(X \mid S_{j}\right)=\sum_{t=1}^{T} \log p\left(X_{t} \mid S_{j}(t)\right)
$$

Closely related to $\mathrm{CE}$, the MMI criterion can then be defined for $\mathrm{X}$ and the reference word string:

$$
L_{M M I}(X, \theta)=-\log \frac{p\left(X \mid \mathrm{S}_{r}\right)^{k} p\left(S_{r}\right)}{\sum_{j} p\left(X \mid \mathrm{S}_{r}\right)^{k} P\left(S_{j}\right)}
$$

These statistics can be obtained efficiently from lattices represented as Weighted Finite State Transducers (WFSTs) using the Forward-Backward algorithm on the Log semiring.

\section{Principles on the Evaluation of Public Servant Execution and Process Management}

Measuring the execution of public servants is an important task for both the government and the society. The measurement standard is similar to the process management issues. A framework highlighting essential building blocks of process management can particularly serve the following purposes: (1) Project management; (2) Vendor Management; (3) Complexity Management; (4) Standards Management; (5) Strategy Management. The recent related researches are concluded in the Figure 2. 


\begin{tabular}{|c|c|c|}
\hline Model & Subject & Source \\
\hline Process Condition Model & $\begin{array}{l}\text { Effectiveness and efficiency } \\
\text { measurement to rate a } \\
\text { process' condition }\end{array}$ & $\begin{array}{l}\text { DeToro and McCabe } \\
\text { (1997) }\end{array}$ \\
\hline $\begin{array}{l}\text { Strategic Alignment Maturity } \\
\text { Model }\end{array}$ & $\begin{array}{l}\text { Maturity of strategic } \\
\text { alignment }\end{array}$ & Luftman (2003) \\
\hline BPR Maturity Model & $\begin{array}{l}\text { Business Process Re- } \\
\text { engineering Programmes }\end{array}$ & Maull et al. (2003) \\
\hline $\begin{array}{l}\text { Harmon's BPM Maturity } \\
\text { Model }\end{array}$ & $\begin{array}{l}\text { BPM maturity model based } \\
\text { on the CMM }\end{array}$ & Harmon $(2003,2004)$ \\
\hline $\begin{array}{l}\text { Rummler-Brache Group's } \\
\text { Process Maturity Model }\end{array}$ & $\begin{array}{l}\text { Success factors for } \\
\text { managing key business } \\
\text { processes }\end{array}$ & $\begin{array}{l}\text { Rummler-Brache } \\
(2004)\end{array}$ \\
\hline OMG's BPM Maturity Model & $\begin{array}{l}\text { Practices applied to the } \\
\text { management of discrete } \\
\text { processes }\end{array}$ & $\begin{array}{l}\text { Curtis et al. (2004); } \\
\text { OMG (2008) }\end{array}$ \\
\hline $\begin{array}{l}\text { Rosemann and de Bruin's } \\
\text { BPM Maturity Model }\end{array}$ & $\begin{array}{l}\text { Maturity of Business } \\
\text { Process Management } \\
\text { capabilities }\end{array}$ & $\begin{array}{l}\text { Rosemann; de Bruin } \\
\text { (2005); de Bruin (2009) }\end{array}$ \\
\hline $\begin{array}{l}\text { Capability Maturity Model } \\
\text { Integration (CMMI) }\end{array}$ & $\begin{array}{l}\text { Maturity of software } \\
\text { development processes }\end{array}$ & SEI (2006a, 2006b) \\
\hline $\begin{array}{l}\text { Hammer's BPM Maturity } \\
\text { Model (Process Audit) }\end{array}$ & $\begin{array}{l}\text { Defining process and } \\
\text { enterprise competencies }\end{array}$ & Hammer (2007) \\
\hline
\end{tabular}

Figure 2.The Related Researches

Through the literature reviews, we could summarize the core elements for evaluating the execution of public servants. Our research distinguishes six core elements critical to the execution of public servants. These are strategic alignment, governance, methods, information technology, people, and culture. The Figure 3 shows the elements.

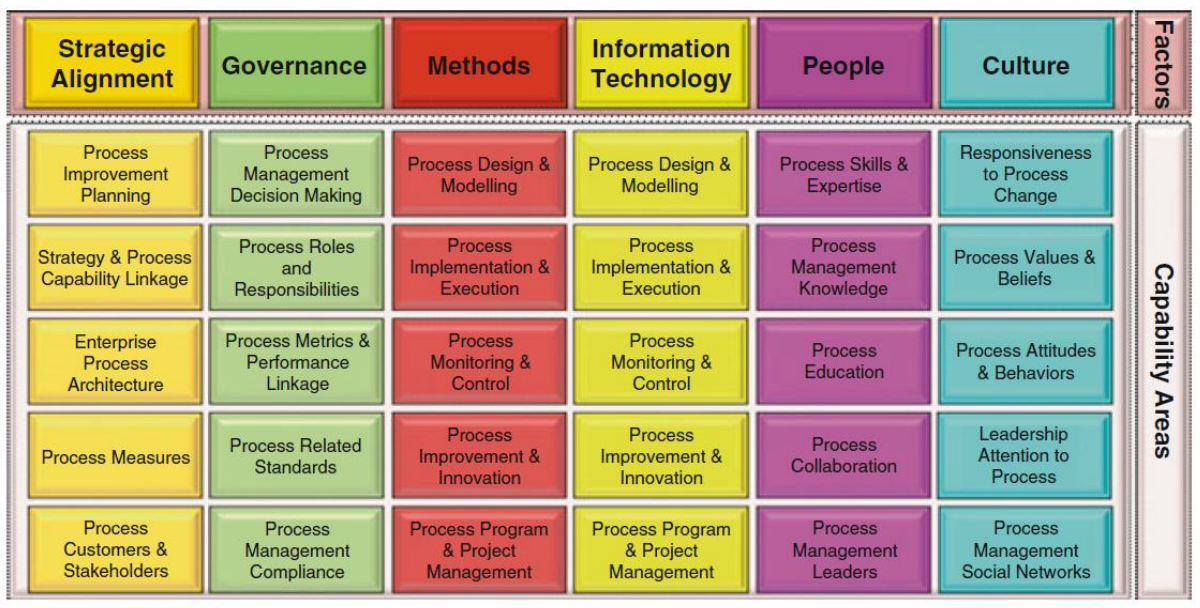

Figure 3.The Core Elements for Measuring

We discuss the core elements in detail. (1) Culture. Culture incorporates the collective values and beliefs in regards to the process-centered organization. Culture is about creating an environment to promote, added a variety of evaluation projects. It need to realize, however, the influence of the cultural activities tend to have a longer range than activities related to the other five factors. (2) People. People at the core of evaluating elements are defined as individuals and groups to improve their process and application and the process management skills and knowledge, in order to improve enterprise performance. (3) Information Technology. IT-based solutions are of significance for initiatives. (4) Methods. Methods in the context of the evaluation are defined as the set of tools and techniques that support and enable activities along the process lifecycle and within 
enterprise-wide BPM initiatives. (5) Governance. A further focus is on the design of decision-making and reward processes to guide process-related actions.

\section{Experimental Analysis and Simulation}

In order to verify the effectiveness and feasibility of our proposed methodology, we conduct numerical and experimental simulation in this Section. We firstly collect data from our pre-tested data set. Then, we use the proposed clustering method to classify the data into clusters for further use. Finally, we evaluate the proposed core elements and analyze the importance of every single element.

\subsection{Set-Up of the Experiment}

The simulation environment is initialized as the follows. Six physical machines equipped with 4 TB hard disk and $6 \mathrm{~GB}$ of RAM, and the simulation software is installed on Windows Win XP platform and Intel core 2 quad core $3.8 \mathrm{GHz}$ and $6 \mathrm{~GB}$ of RAM. To compare the advantages of the candidate clustering algorithms, we take three other algorithms as the reference.

\subsection{Data Clustering Experiment}

The numerical result is shown in the Table 3 and in the Figure 4 and we draw the curve for the data. We could instantly see from the Table and the Figure that our proposed cluster method out-performs compared with the other methods.

Table 3.The Data Clustering Experiment Result

\begin{tabular}{c|c|c|c|c}
\hline Number & Our Method & FCM & EM & K-means \\
\hline $\mathbf{1}$ & 89.6 & 85.6 & 86.5 & 86.6 \\
\hline $\mathbf{2}$ & 91.3 & 88.3 & 85.4 & 84.1 \\
\hline $\mathbf{3}$ & 93.4 & 91.0 & 88.8 & 90.5 \\
\hline $\mathbf{4}$ & 90.5 & 78.3 & 86.5 & 88.6 \\
\hline $\mathbf{5}$ & 89.1 & 80.5 & 82.6 & 87.1 \\
\hline $\mathbf{6}$ & 95.3 & 90.4 & 91.1 & 89.2 \\
\hline $\mathbf{7}$ & 89.1 & 88.8 & 87.2 & 86.3 \\
\hline $\mathbf{8}$ & 90.2 & 92.1 & 88.4 & 88.1 \\
\hline
\end{tabular}

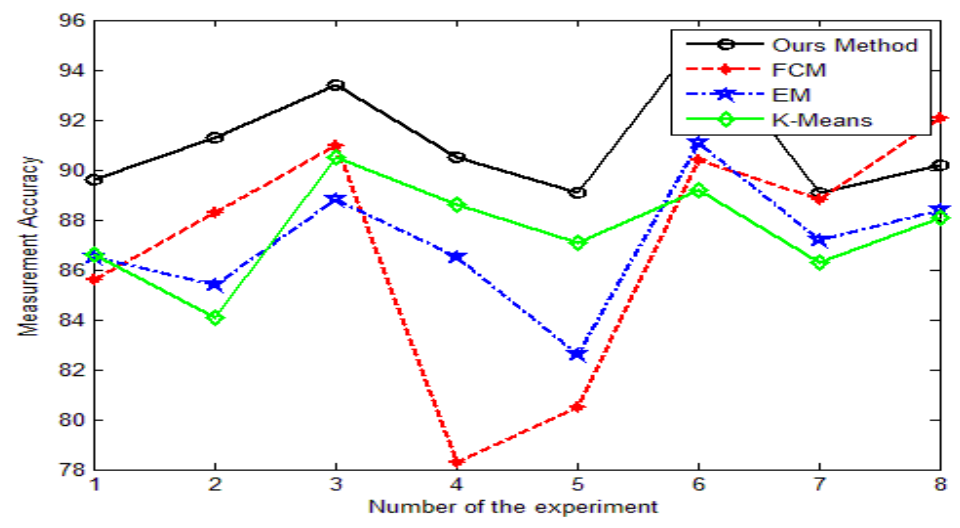

Figure 4. The Comparison Result for the Clustering Methods 


\subsection{Experimental Analysis on Evaluation of Public Servant Execution}

In this experiment, we record the weight of different elements for execution of public servants. The detailed data is shown in the Table 4. We conducted the experiment for 20 times to find out for the most important influential element for evaluating of the servants. From the experimental result, we conclude that the element of people is the most important factor for the execution of public servants.

Table 4. The Experimental Analysis on Evaluation of Public Servant Execution

\begin{tabular}{c|c|c|c|c|c}
\hline Number(Weight) & Culture & People & IT & Methods & Governance \\
\hline $\mathbf{1}$ & 11 & 24 & 21 & 19 & 22 \\
\hline $\mathbf{2}$ & 12 & 26 & 19 & 14 & 21 \\
\hline $\mathbf{3}$ & 11 & 27 & 18 & 17 & 21 \\
\hline $\mathbf{4}$ & 9 & 21 & 21 & 18 & 24 \\
\hline $\mathbf{5}$ & 13 & 19 & 24 & 14 & 22 \\
\hline $\mathbf{6}$ & 11 & 22 & 20 & 16 & 19 \\
\hline $\mathbf{7}$ & 6 & 18 & 15 & 15 & 20 \\
\hline $\mathbf{8}$ & 8 & 27 & 20 & 19 & 21 \\
\hline $\mathbf{9}$ & 15 & 26 & 22 & 21 & 22 \\
\hline $\mathbf{1 0}$ & 12 & 22 & 21 & 22 & 21 \\
\hline $\mathbf{1 1}$ & 11 & 26 & 22 & 13 & 25 \\
\hline $\mathbf{1 2}$ & 16 & 23 & 24 & 14 & 16 \\
\hline $\mathbf{1 3}$ & 9 & 21 & 16 & 17 & 15 \\
\hline $\mathbf{1 4}$ & 4 & 24 & 21 & 11 & 19 \\
\hline $\mathbf{1 5}$ & 17 & 26 & 20 & 21 & 21 \\
\hline $\mathbf{1 6}$ & 2 & 29 & 24 & 24 & 18 \\
\hline $\mathbf{1 7}$ & 6 & 19 & 16 & 13 & 20 \\
\hline $\mathbf{1 8}$ & 5 & 21 & 26 & 21 & 20 \\
\hline $\mathbf{1 9}$ & 13 & 25 & 21 & 20 & 22 \\
\hline $\mathbf{2 0}$ & 8 & 22 & 17 & 15 & 21 \\
\hline
\end{tabular}

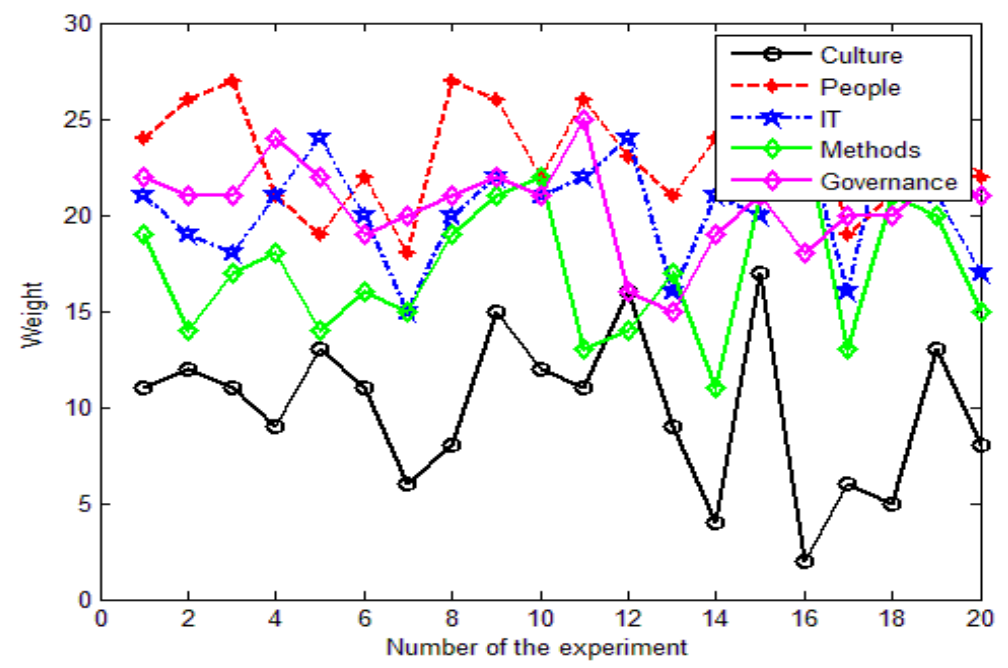

Figure 5. The Comparison Result for Evaluation of Public Servant Execution 


\section{Conclusion and Summary}

Data mining modelling techniques have emerged and rapidly developed as an alternative powerful meta-learning tool to accurately and fast analyze the massive volume of data generated by modern applications. In this paper, we firstly analyze based on the traditional algorithm on evaluation of public servant execution shortcomings which holds lots of drawbacks such as grass-roots public servants often rely on past experience and not to learn new knowledge and master new technology which has caused many originally can do good things to do so. In the transformation type study, objective is to learn to observe, specific examples to specific examples from the same distribution. In this paper, we conduct research on execution of public servants based on data mining technique and joint modeling analysis of multiple factors under big data environment. Firstly, we introduce some state-of-the-art clustering algorithm to serve as the basis of our model. Combined with deep neural network and optimization modelling, we propose our support vector machine based data clustering algorithm through multiple factor modelling. Subsequently, we discuss the principles on evaluation of public servant execution and process management. In the experimental part, we conduct experiment on both data clustering based data pre-processing step and the evaluation of elements' weight for process management. The result indicates the most important factor for management and the feasibility and effectiveness of our proposed clustering method. Execution of public servants at the grass-roots level directly affects the realization of policy goal and therefore, must raise its execution. To solve these problems of execution in terms of grass-roots public servants, improve its execution, we need in such aspects as concept, skills, and institutional mechanisms to find a suitable way. The way we put forward the perfect solved the problem.

In the near future, we plan to do more theoretical analysis on the data clustering part. Combination of more deep neural network structures and optimization approaches will be the core area of our future research. We believe the application of our method will have more related topics to be researched such as enhancing the sense of responsibility and the efficiency.

\section{Acknowledgement}

Fund Projects: general program supported by the national fund of social science (14BKS032); youth program supported by social science fund of Heilongjiang Province (12C065); general program supported by Heilongjiang provincial natural science fund (G2015008)

\section{References}

[1] C. Anyela, D. Papadopoulou, Z. Spyropoulou, K. Vlachonasios, J. H. Doonan and Alan P. Gay, "Objective definition of rosette shape variation using a combined computer vision and data mining approach”, PloS one 9, no. 5, (2014), e96889.

[2] B. Vishwanath, V. Kumar, P. Kumari and J. Pascual, "KNN based Machine Learning Approach for Text and Document Mining”, International Journal of Database Theory and Application 7, no. 1, (2014), pp. 61-70.

[3] Y. Erman, S. Ozekes and Y. K. Türel, "Predicting dropout student: An application of data mining methods in an online education program", European Journal of Open, Distance and e-Learning 17, no. 1, (2014), pp. 118-133.

[4] W. Frederik, F. Delmar and A. R. Johnson, "Entrepreneurship in the Age of Big Data: A Researcher's Guide to Data Mining", Inference and Prediction, (2015).

[5] X. Jian, T. L. Wickramarathne, N. V. Chawla, E. K. Grey, K. Steinhaeuser, R. P. Keller, J. M. Drake and D. M. Lodge, "Improving management of aquatic invasions by integrating shipping network, ecological, and environmental data: Data mining for social good", In Proceedings of the 20th ACM SIGKDD international conference on Knowledge discovery and data mining, pp. 1699-1708. ACM, (2014).

[6] W. Xindong, X. Zhu, G. Q. Wu and W. Ding, "Data mining with big data", Knowledge and Data Engineering, IEEE Transactions on 26, no. 1, (2014), pp. 97-107. 
[7] E. Robert, T. Werge, L. J. Jensen and S. Brunak, "Dose-specific adverse drug reaction identification in electronic patient records: temporal data mining in an inpatient psychiatric population", Drug Safety, vol. 37, no. 4, (2014), pp. 237-247.

[8] S. Dóra, S. V. Landeghem, K. Baerenfaller, L. Baeyens, J. Blomme, R. C. Sáez and S. D. Bodt, "The Known Leaf literature duration system captures knowledge about Arabidopsis leaf growth and development and facilitates integrated data mining”, Current Plant Biology, (2015).

[9] Z. Jian and L. Shen, "An Improved Fuzzy c-Means Clustering Algorithm Based on Shadowed Sets and PSO", Computational intelligence and neuroscience 2014, (2014).

[10] Chandrasekar P. and M. Krishnamoorthi, "BHOHS: A Two Stage Novel Algorithm for Data Clustering", In Intelligent Computing Applications (ICICA), 2014 International Conference on, IEEE, (2014), pp. $138-142$.

[11] K. Ganesh, A. J. Kulkarni and R. Paramesran, "A hybrid approach for data clustering based on modified cohort intelligence and K-means", Expert Systems with Applications 41, no. 13, (2014), pp. 6009-6016.

[12] L. P. Lan, P. W. Huang, C. H. Kuo and Y. H. Lai, "A size-insensitive integrity-based fuzzy c-means method for data clustering", Pattern Recognition 47, no. 5, (2014), pp. 2042-2056.

[13] Q. Hongwu, X. Ma, T. Herawan and J. M. Zain, "MGR: An information theory based hierarchical divisive clustering algorithm for categorical data", Knowledge-Based Systems, vol. 67, (2014), pp. 401411.

[14] K. C. Maria, A. Ławrynowicz, C. d'Amato, A. Kalousis, P. Nguyen, R. Palma, R. Stevens and M. Hilario, "The Data Mining Optimization Ontology", Web Semantics: Science, Services and Agents on the World Wide Web, (2015).

[15] T. Olivier and O. Pourquié, "Manteia, a predictive data mining system for vertebrate genes and its applications to human genetic diseases", Nucleic acids research 42, no. D1, D882-D891, (2014).

[16] Sosa C. M., M. A. Pavarotti, M. N. Zanetti, F. C. M. Zoppino, G. A. De Blas and L. S. Mayorga "Kinetics of human sperm acrosomal exocytosis", Molecular human reproduction, gau110, (2014).

[17] G. D. Sunayana, M. A. Ardeshana and S. D. Bhatt, "Advances in Em (Expectation-Maximization) Algorithm for Image Classification as Per Cost and Accuracy", IJRCCT 3, no. 1, (2014), pp. 065-069.

[18] W. Zhang, S. M. Liu, D. Wei, Z. J. Wang, M. L. Yang and Y. Li, "Probabilistic wind power forecast using sparse Bayesian learning of unified kernel function", In Transportation Electrification Asia-Pacific (ITEC Asia-Pacific), 2014 IEEE Conference and Expo, IEEE, (2014), pp. 1-4.

[19] W. Pan, Z. Lu, J. Hu and C. Zhou, "Sensitivity analysis of the variance contributions with respect to the distribution parameters by the kernel function", Computers \& Mathematics with Applications, vol. 67, no. 10, (2014), pp. 1756-1771.

[20] Y. Yun and J. Jiang, "HMM-based hybrid meta-clustering ensemble for temporal data", KnowledgeBased Systems, vol. 56, (2014), pp. 299-310.

[21] Z. Jian and L. Shen, "An Improved Fuzzy c-Means Clustering Algorithm Based on Shadowed Sets and PSO", Computational intelligence and neuroscience 2014, (2014).

[22] S. Nabil, O. A. Arqub and S. Momani, "Analytical solution of nonlinear second-order periodic boundary value problem using reproducing kernel method", Journal of Computational Analysis and Applications, vol. 16, no. 4, (2014), pp. 750-762.

[23] N. Z. Mohd, A. K. Junoh and A. K. Ariffin, "Vehicle interior noise and vibration level assessment through the data clustering and hybrid classification model", Applied Acoustics, vol. 87, (2015), pp. 922.

[24] P. M. Reza, S. F. Hafshejani and L. Shirvani, "Complexity of interior-point methods for linear optimization based on a new trigonometric kernel function", Journal of Computational and Applied Mathematics, vol. 255, (2014), pp. 74-85.

[25] W. P. An, X. S. Gan and W. M. Gao, "Research on Nonlinear Modeling Method of Support Vector Machine with Wavelet Derivation Kernel Function", In Applied Mechanics and Materials, vol. 687, (2014), pp. 1408-1411.

[26] S. Sainbayar and R. Fergus, "Learning from Noisy Labels with Deep Neural Networks", arXiv preprint arXiv: 1406.2080, (2014).

[27] F. Haoqiang, Z. Cao, Y. Jiang, Q. Yin and C. Doudou, "Learning deep face representation", arXiv preprint arXiv:1403.2802, (2014).

[28] O'Brien, Will and J. Sarkis, "The potential of community-based sustainability projects for deep learning initiatives", Journal of Cleaner Production, vol. 62, (2014), pp. 48-61.

[29] S. Yi, Y. Chen, X. Wang and X. Tang, "Deep learning face representation by joint identificationverification", In Advances in Neural Information Processing Systems, (2014), pp. 1988-1996.

[30] H. Tomoyuki, K. Hanawa and U. Yamamoto, "Gender classification of subjects from cerebral blood flow changes using Deep Learning", In Computational Intelligence and Data Mining (CIDM), 2014 IEEE Symposium on, IEEE, (2014), pp. 229-233. 
International Journal of Database Theory and Application Vol.9, No.1 (2016) 